\title{
Broadband optical chaos for stimulated Brillouin scattering suppression in power over fiber
}

\author{
Xuelei Fu, ${ }^{1}$ Sze-Chun Chan, ${ }^{1, *}$ Qing Liu, ${ }^{1}$ and Kenneth Kin-Yip Wong ${ }^{2}$ \\ ${ }^{1}$ Department of Electronic Engineering, City University of Hong Kong, Hong Kong, China \\ ${ }^{2}$ Department of Electrical and Electronic Engineering, University of Hong Kong, Hong Kong, China \\ ${ }^{*}$ Corresponding author: scchan @ cityu.edu.hk
}

Received 2 March 2011; revised 28 June 2011; accepted 5 July 2011; posted 11 July 2011 (Doc. ID 143478); published 21 July 2011

\begin{abstract}
Broadband chaos generated in an optically injected semiconductor laser is applied for power-over-fiber transmission. By varying the injection power, period-one oscillation, period-two oscillation, and chaotic oscillation are observed in the injected slave laser, indicating a period-doubling route to chaos. Compared to the free-running output of the laser, its chaotic output has a drastically increased signal bandwidth, which leads to a $19 \mathrm{~dB}$ increase of the stimulated Brillouin scattering threshold. Using a chaos of $5.2 \mathrm{GHz}$ bandwidth, a maximum optical power of $27 \mathrm{dBm}$ is obtained after $20 \mathrm{~km}$ transmission over fiber, which is applicable to optically powering some advanced communication networks. The approach uses the inherent nonlinear laser dynamics, which requires no modulation electronics or microwave signal sources. (c) 2011 Optical Society of America
\end{abstract}

OCIS codes: $\quad 140.5960,140.3520,140.1540,290.5900$.

\section{Introduction}

Power over fiber $(\mathrm{PoF})$ is a technique that uses optical fiber for power distribution. In a PoF system, optical power generated by a high-power light source is coupled into an optical fiber and transmitted to the remote site for optoelectronic conversion using photovoltaics (PVs). Compared to traditional copperwire power supply systems, $\mathrm{PoF}$ systems provide excellent isolation, low distributed loss, and immunity to electromagnetic noise. With the advances of lowpower devices, PoF has found applications in sensor networks, data links in high-voltage environments, and integration with the currently existing fiber communication networks and radio-over-fiber systems [1-5]. Although multimode fibers are often applied for $\mathrm{PoF}$, single-mode fibers are sometimes preferred. The main advantage of single-mode fibers for $\mathrm{PoF}$ is their relatively low bending loss [6]. Single-mode fibers are also compatible with most deployed highspeed data networks and sensing devices $[1,2]$.

0003-6935/11/250E92-05\$15.00/0

(C) 2011 Optical Society of America
The key limitation on the power transfer capacity of single-mode fibers is caused by stimulated Brillouin scattering (SBS). This nonlinear optical process generates a backward-propagating Stokes wave as a reflection that limits the net incident power at the input end to the SBS threshold [ㅁ-12]:

$$
P_{p}^{\text {th }}=\left(1+\frac{\Delta \nu_{p}}{\Delta f_{B}}\right) \cdot \frac{\ln G_{B}^{\text {th }} A_{\text {eff }} K}{\tilde{g}_{B 0}^{\max } l_{\mathrm{eff}}},
$$

where $\Delta f_{B}$ is the FWHM linewidth of the Brillouin gain spectrum, $\Delta \nu_{p}$ is the FWHM linewidth of the pump wave, $G_{B}^{\text {th }}$ is the threshold amplification factor, $A_{\text {eff }}$ is the effective mode area, $\tilde{g}_{B 0}^{\max }$ is the peak Brillouin gain factor for an idealized cw pump, $l_{\text {eff }}=$ $(1-\exp (-\alpha l)) / \alpha$ is the effective length of the fiber of physical length $l$ and attenuation $\alpha$, and $K$ is the polarization factor that accounts for polarization scrambling in typical single-mode fibers with residual fluctuating birefringence. Both the gain and the pump line shapes are assumed to be Lorentzian.

Once a fiber is chosen, the only way to raise $P_{p}^{\text {th }}$ is to increase the pump wave linewidth $\Delta \nu_{p}$. A possibility is to employ high-power multimode laser diodes 
as the light source [13]. However, the coupling loss into the fiber is large for multitransverse mode lasers. The multilongitudinal mode emission also takes up a large wavelength band, which causes difficulties multiplexing with data. A recent solution employed a single-mode laser that was modulated externally and amplified optically [14,15]. The method used external modulators together with highspeed driving electronics to broaden $\Delta \nu_{p}$. Related methods often require extra dithering electronics, multitone microwave sources, high-speed pseudorandom bit generators, or strongly amplified microwave noise sources $[16,17]$.

In this paper, broadband optical chaos generated from a single-mode semiconductor laser using optical injection is applied for PoF. A significant increment in the bandwidth $\Delta \nu_{p}$ of chaos, as compared to that of the cw output from the free-running laser, drastically raises the SBS threshold and, thus, increases the maximum power deliverable over fibers. An increment of the SBS threshold by $19 \mathrm{~dB}$ is achieved and is comparable to that reported using noisy external modulation [17]. Moreover, since chaotic oscillation is part of the inherent laser nonlinear dynamics [18-21], our approach requires no external modulators, dithering circuitries, or high-speed electronics. The optical spectrum generated from the chaotic single-mode laser is continuous, which leads to better bandwidth efficiency as compared to conventional PoF using multimode lasers [13].

\section{Experimental Setup}

Figure 1 shows the schematic of the setup. The lasers used are distributed-feedback lasers (Mitsubishi ML920T43S-01) emitting at $1548 \mathrm{~nm}$. Light from the master laser is injected into the slave laser through a free-space optical circulator, which ensures unidirectional injection. The circulator is arranged such that the polarizations of the injection and the slave laser are the same, thereby minimizing the injection power required to generate chaos [20]. The master laser is biased at $133.4 \mathrm{~mA}$ and temperature stabilized at $18.00^{\circ} \mathrm{C}$, while the slave laser is biased at $40.0 \mathrm{~mA}$ and temperature stabilized at $27.00^{\circ} \mathrm{C}$. Under this operating condition, the detuning frequency of the master laser from the slave laser is $11.0 \mathrm{GHz}$. The injection power can be tuned by adjusting a

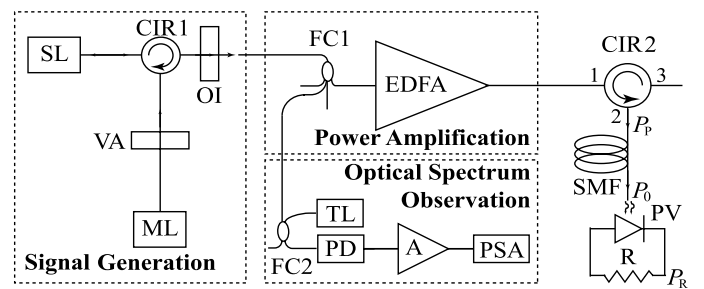

Fig. 1. Schematic of PoF transmission using optical chaos. ML, master laser; SL, slave laser; VA, variable attenuator; CIR, circulator; OI, optical isolator; FC, coupler; EDFA, erbium-doped fiber amplifier; SMF, single-mode fiber; PV, photovoltaic converter; $\mathrm{R}$, resistor; TL, tunable laser; $\mathrm{PD}$, photodetector; A, microwave amplifier; PSA, power spectrum analyzer. variable attenuator. The slave laser has a relaxation resonance frequency of about $11 \mathrm{GHz}$. The output power is about $10 \mathrm{~mW}$ irrespective of the dynamics. Its threshold current is $9 \mathrm{~mA}$. An optical isolator is adopted to prevent any backreflection.

The output after the optical isolator is coupled to a fiber coupler. One branch is directed to an erbiumdoped fiber amplifier (EDFA, consisting of cascading Amonics AEDFA-23-B-FA and IPG Photonics EAR10k-C), which can boost the optical power up to $36 \mathrm{dBm}$. A fiber-based optical circulator is employed to protect the EDFA from any possible reflected power. Single-mode fibers (Corning SMF-28e+) of lengths $l=5,10,15$, and $20 \mathrm{~km}$ are adopted to demonstrate long-distance power transmission. The fibers have attenuation $\alpha=0.046 \mathrm{~km}^{-1}$ and Brillouin linewidth $\Delta f_{B}=25 \mathrm{MHz}$ [22]. At the output end of the fiber, electrical power is obtained through optoelectronic conversion using a PV converter (JDSU 9LC0097), which drives a load resistor of $353 \Omega$. The resistance is optimized for maximum conversion efficiency.

Since traditional optical spectrum analyzers cannot provide a fine enough frequency resolution, a specially constructed system is applied for optical spectrum observation. As shown in Fig. 1, a branch of the generated signal is combined with a narrowlinewidth cw emission from a tunable laser (HP $8168 \mathrm{~A}$ ) by a $3 \mathrm{~dB}$ fiber coupler. The wavelength of the tunable laser is fixed at $0.03 \mathrm{~nm}$ shorter than that of the master laser. The beat signal is then detected by a $43 \mathrm{GHz}$ photodetector (Newport $\mathrm{AD}-10 \mathrm{ir}$ ), amplified by $40 \mathrm{~dB}$ using two cascaded microwave amplifiers (HP 83006A and HP 83017A), and monitored on a $26.5 \mathrm{GHz}$ power spectrum analyzer (Agilent N9010A).

\section{Experimental Results}

The optical spectrum from the slave laser is obtained and plotted in Fig. 2. The frequency axis is offset to the free-running frequency of the slave laser. The arrows indicate regeneration of the optical injection. By varying the injection power, different nonlinear dynamical states are observed from the slave laser. When the laser is free-running in Fig. 2(a), it emits single-frequency $\mathrm{cw}$ light. The linewidth is measured to be $\Delta \nu_{p}=5 \mathrm{MHz}$. When the injection power is $0.05 \mathrm{~mW}$, as in Fig. 2(b), the slave laser is forced into period-one oscillation, generating two components separated by $11.1 \mathrm{GHz}$. When the injection power is increased to $0.21 \mathrm{~mW}$ in Fig. 2(c), period-two oscillation is observed with generation of a subharmonic through period-doubling bifurcation. When the injection power increases to $0.31 \mathrm{~mW}$ in Fig. 2(d), optical chaos is clearly observed through a period-doubling route to chaos [18]. The spectrum shows significant broadening of signal bandwidth due to chaotic oscillation. As an estimation, by fitting the chaotic spectrum to a Lorentzian function, the linewidth of the signal is estimated to be $\Delta \nu_{p}=5.2 \mathrm{GHz}$, which is much broader than the Brillouin linewidth. It is 


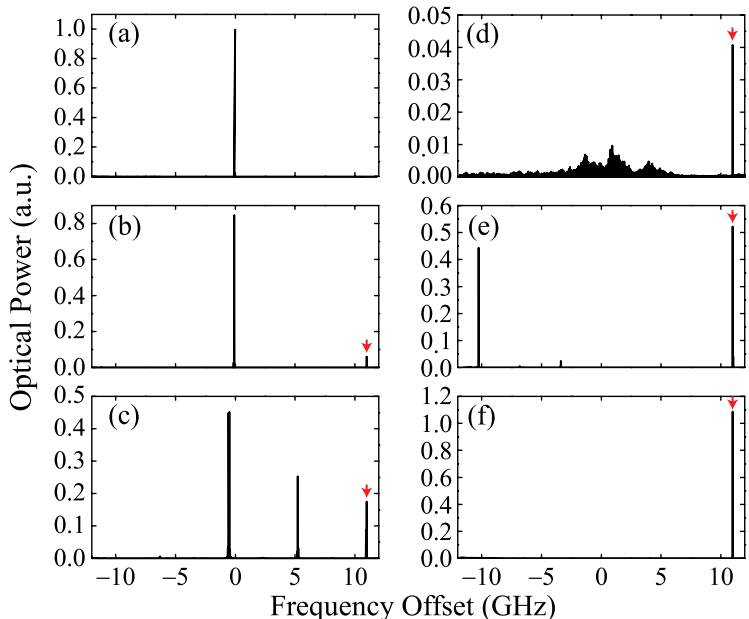

Fig. 2. (Color online) Optical spectra from the slave laser in the (a) free-running state, (b) period-one state, (c) period-two state, (d) chaotic state, (e) period-one state, and (f) stable-locking state, where the injection power is $0,0.05,0.21,0.31,3.78$, and $23.6 \mathrm{~mW}$, respectively. The injection detuning frequency is kept at $11.0 \mathrm{GHz}$, as the arrows indicate.

important to note that the regeneration is only 0.04 as strong as the free-running emission in Fig. 2(a). Most of the power in Fig. 2(d) is carried by the broadband chaos. The chaotic spectrum remains unchanged as long as the bias currents and the temperatures of the lasers are kept constant. When the injection is increased to $3.78 \mathrm{~mW}$ in Fig. 2(e), the slave laser goes back to period-one oscillation at a different frequency separation of $21.2 \mathrm{GHz}$. When the injection is $23.6 \mathrm{~mW}$ in Fig. $2(\mathrm{f})$, the slave laser is finally stably locked by the master laser [20]. In the following, the chaotic state in Fig. 2(d) is applied for $\mathrm{PoF}$, which is compared with the performance of the cw emission from the free-running state in Fig. 2(a).

The output optical power $P_{0}$ after PoF transmission is plotted against the input power $P_{p}$ in Fig. $\underline{3}$ with different fiber lengths. The results using the chaotic and the free-running states are respectively obtained as closed and open symbols for comparison. For the free-running state, the output power quickly

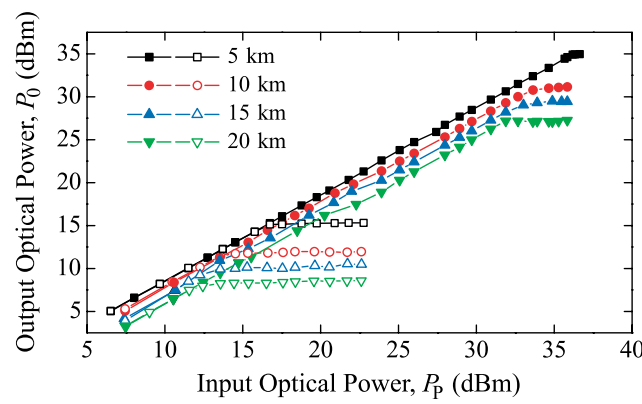

Fig. 3. (Color online) Output optical power versus input optical power for transmission over fibers. The fiber lengths are indicated by the legends. The open and closed symbols are obtained when the slave laser is in the free-running state and the chaotic state, respectively. For each curve, the output power saturates because of SBS reflection.

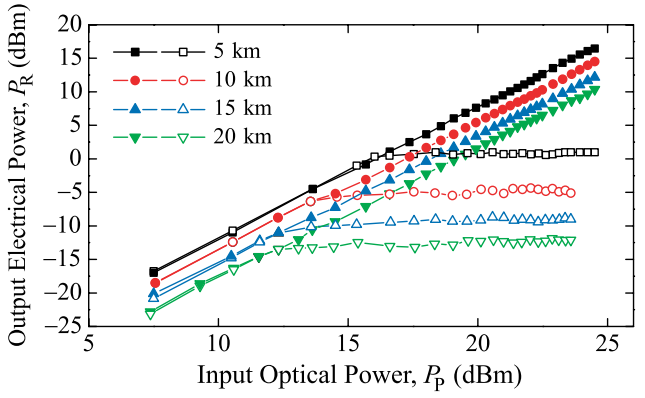

Fig. 4. (Color online) Output electrical power versus input optical power for transmission over fibers. The fiber lengths are indicated by the legends. The open and closed symbols are obtained when the slave laser is in the free-running state and the chaotic state, respectively.

saturates due to SBS, which is confirmed by monitoring the spectrum of the reflected signal from the fiber. For the chaotic state, the saturated power is significantly raised due to the broadened linewidth. Taking $20 \mathrm{~km}$ transmission as an example, the output optical power is saturated at $8.6 \mathrm{dBm}$ for the freerunning state, while the saturated power is greatly increased to $27 \mathrm{dBm}$ using the chaotic state.

Moreover, Fig. 4 shows the associated electrical power $P_{R}$ after optoelectronic conversion using the PV converter for demonstration. The performance of the PV converter limits the input power to only $24.5 \mathrm{dBm}$ in order to prevent damage. Nonetheless, the electrical power obtained over a $5 \mathrm{~km}$ fiber using the free-running state saturates at $0.8 \mathrm{dBm}$ due to SBS, while at least $16.4 \mathrm{dBm}$ of electrical power is obtained using the chaotic state. This electrical power level is only limited by the PV converter used, but it is already adequate for powering some advanced remote units [4].

To further characterize the system, the saturated output optical power for the free-running and chaotic states is plotted in Fig. 5 . The data points are fit to $\exp (-\alpha l) P_{p}^{\text {th }}$ using Eq. $(\underline{\overline{1}})$. The saturated power for the chaotic state increases by $19 \mathrm{~dB}$ as compared to the free-running state. The increment is a bit less than the estimated $22 \mathrm{~dB}$ from the measurements of linewidths $\Delta \nu_{p}$ in Fig. 2 . This is possibly due to overestimation of the linewidth of the chaotic signal when fitting to a Lorentzian function. In the experiments,

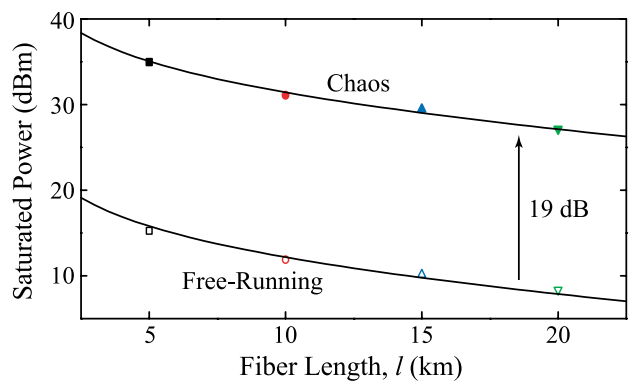

Fig. 5. (Color online) Saturated output optical power versus fiber length. The open and closed symbols are obtained when the slave laser is in the free-running state and the chaotic state, respectively. 

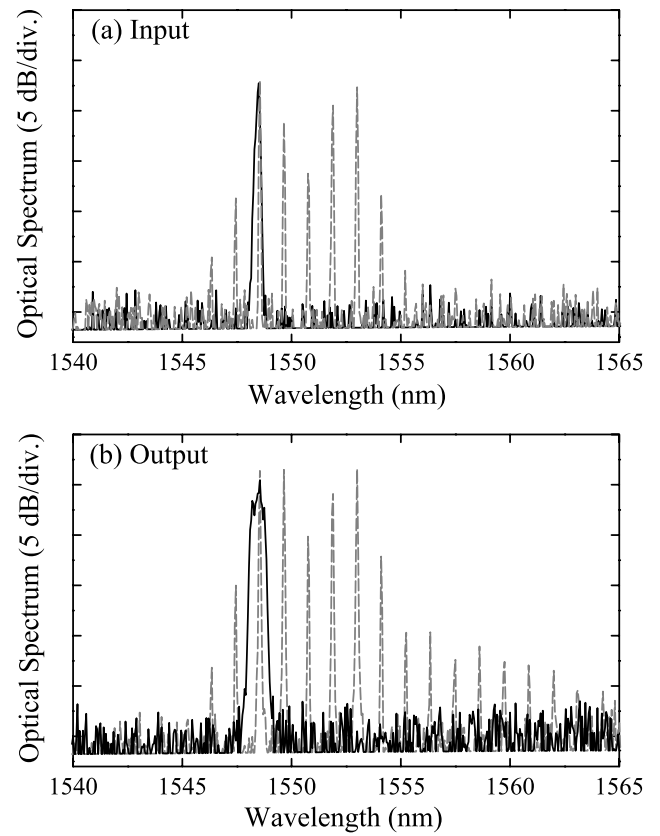

Fig. 6. Optical spectra measured (a) at the input to the EDFA and (b) at the output after PoF transmission. The solid curves are obtained when the optically injected single-mode laser is in the chaotic state. The dashed curves show the spectra obtained when the laser is replaced by a cw multilongitudinal mode laser without optical injection.

the saturated power does not depend on the state of polarization at the input of the fiber, which is due to polarization scrambling by residual fluctuating birefringence in the fiber [10].

\section{Discussion}

When compared to a conventional cw multilongitudinal mode laser, the chaotic single-mode laser is more bandwidth efficient. In Fig. 6, the solid and dashed curves are the optical spectra from the chaotic single-mode laser under optical injection and from a cw multimode laser without optical injection, respectively. Figure 6(a) shows the spectra measured at the input to the EDFA. Chaotic emission from the singlemode laser is concentrated at a single peak at about $1548 \mathrm{~nm}$, while cw emission from the multimode laser is distributed over several peaks and occupies a wide bandwidth. Furthermore, the signals are transmitted over fiber for $20 \mathrm{~km}$ after being amplified by the EDFA to $31 \mathrm{dBm}$, which is below the SBS thresholds for both signals. Because of Kerr nonlinearity, spectral broadening for both signals is observed, as Fig. $6(\mathrm{~b})$ shows. The discrete peaks of the dashed curve from the multimode laser are frequency mixed and result in a significant overall broadening to about $20 \mathrm{~nm}$. By contrast, the solid curve for the chaotic single-mode laser is only slightly broadened, where the measured $20 \mathrm{~dB}$ bandwidth is just about $1 \mathrm{~nm}$. Thus, the smaller overall bandwidth of the chaotic single-mode laser is attributed to its continuous spectrum centered at a single mode.

\section{Conclusion}

Optical chaos generated by optically injecting a semiconductor laser is applied for suppressing SBS in PoF. Because of the significantly increased signal bandwidth of the chaotic state, the saturated power for $\mathrm{PoF}$ is increased by $19 \mathrm{~dB}$. A maximum optical power of $27 \mathrm{dBm}$ is received after $20 \mathrm{~km}$ transmission. The approach uses the inherent nonlinear laser dynamics, which requires no modulation electronics or microwave signal sources. It is a promising candidate for optically powered sensing and communication networks. Chaos generation using simplified methods like optical feedback can also be considered.

The work described in this paper was fully supported by a grant from City University of Hong Kong (Project No. 7002448) and a grant from the Research Grant Council of Hong Kong, China (Project No. CityU 111210).

\section{References}

1. M. Roeger, G. Boettger, M. Dreschmann, C. Klamouris, M. Huebner, A. W. Bett, J. Becker, W. Freude, and J. Leuthold, "Optically powered fiber networks," Opt. Express 16, 21821-21834 (2008).

2. Y. Tanaka, T. Shioda, T. Kurokawa, J. Oka, K. Ueta, and T. Fukuoka, "Power line monitoring system using fiber optic power supply," Opt. Rev. 16, 257-261 (2009).

3. G. Boettger, M. Dreschmann, C. Klamouris, M. Huebner, M. Roeger, A. W. Bett, T. Kueng, J. Becker, W. Freude, and J. Leuthold, "An optically powered video camera link," IEEE Photon. Technol. Lett. 20, 39-41 (2008).

4. D. Wake, A. Nkansah, and N. Gomes, "Optical powering of remote units for radio over fiber links," in IEEE International Topical Meeting on Microwave Photonics (IEEE, 2007), pp. 29-32.

5. D. Wake, A. Nkansah, N. J. Gomes, C. Lethien, C. Sion, and J.-P. Vilcot, "Optically powered remote units for radio-overfiber systems," J. Lightwave Technol. 26, 2484-2491 (2008).

6. T. C. Banwell, R. C. Estes, L. A. Reith, P. W. Shumate, Jr., and E. M. Vogel, "Powering the fiber loop optically-a cost analysis," J. Lightwave Technol. 11, 481-494 (1993).

7. E. P. Ippen and R. H. Stolen, "Stimulated Brillouin scattering in optical fibers," Appl. Phys. Lett. 21, 539-541 (1972).

8. J. M. Liu, Photonic Devices (Cambridge University, 2005).

9. A. B. Ruffin, M. J. Li, X. Chen, A. Kobyakov, and F. Annunziata, "Brillouin gain analysis for fibers with different refractive indices," Opt. Lett. 30, 3123-3125 (2005).

10. R. H. Stolen, "Polarization effects in fiber Raman and Brillouin lasers," IEEE J. Quantum Electron. 15, 1157-1160 (1979).

11. Y. Aoki, K. Tajima, and I. Mito, "Input power limits of singlemode optical fibers due to stimulated Brillouin scattering in optical communication systems," J. Lightwave Technol. 6, 710-719 (1988).

12. M. O. van Deventer and A. J. Boot, "Polarization properties of stimulated Brillouin scattering in single-mode fibers," J. Lightwave Technol. 12, 585-590 (1994).

13. H. Miyakawa, Y. Tanaka, and T. Kurokawa, "Design approaches to power-over-optical local-area-network systems," Appl. Opt. 43, 1379-1389 (2004).

14. S. Yang, X. Xu, Y. Zhou, K. K. Y. Cheung, and K. K. Y. Wong, "Continuous-wave single-longitudinal-mode fiber-optical parametric oscillator with reduced pump threshold," IEEE Photon. Technol. Lett. 21, 1870-1872 (2009). 
15. X. Xu, K. K. Y. Cheung, S. Yang, Y. Liang, T. I. Yuk, and K. K. Y. Wong, "Optically powered WDM signal transmission system with distributed parametric amplification," IEEE Photon. Technol. Lett. 22, 1232-1234 (2010).

16. T. Torounidis, B. E. Olsson, H. Sunnerud, M. Karlsson, and P. A. Andrekson, "Fiber-optic parametric amplifier in a loop mirror configuration," IEEE Photon. Technol. Lett. 17, 321-323 (2005).

17. J. B. Coles, B. P. P. Kuo, N. Alic, S. Moro, C. S. Bres, J. M. Chavez Boggio, P. A. Andrekson, M. Karlsson, and S. Radic, "Bandwidth-efficient phase modulation techniques for stimulated Brillouin scattering suppression in fiber optic parametric amplifiers," Opt. Express 18, 18138-18150 (2010).

18. T. B. Simpson, J. M. Liu, A. Gavrielides, V. Kovanis, and P. M. Alsing, "Period-doubling route to chaos in a semiconductor laser subject to optical injection," Appl. Phys. Lett. 64, 3539-3541 (1994).

19. F. Y. Lin and J. M. Liu, "Harmonic frequency locking in a semiconductor laser with delayed negative optoelectronic feedback," Appl. Phys. Lett. 81, 3128-3130 (2002).

20. S. C. Chan, S. K. Hwang, and J. M. Liu, "Period-one oscillation for photonic microwave transmission using an optically injected semicondutor laser," Opt. Express 15, 14921-14935 (2007).

21. Y. Takeuchi, R. Shogenji, and J. Ohtsubo, "Chaotic dynamics in semiconductor lasers subjected to polarization-rotated optical feedback," Appl. Phys. Lett. 93, 181105 (2008).

22. A. Kobyakov, M. Sauer, and D. Chowdhury, "Stimulated Brillouin scattering in optical fibers," Adv. Opt. Photon. 2, 1-59 (2010). 